\title{
Uniform Parallel-Machine Scheduling with Time Dependent Processing Times
}

\author{
Juan Zou • Yuzhong Zhang • Cuixia Miao
}

Received: 16 January 2013 / Revised: 9 April 2013 / Accepted: 18 April 2013 /

Published online: 24 May 2013

(C) Operations Research Society of China, Periodicals Agency of Shanghai University, and

Springer-Verlag Berlin Heidelberg 2013

\begin{abstract}
We consider several uniform parallel-machine scheduling problems in which the processing time of a job is a linear increasing function of its starting time. The objectives are to minimize the total completion time of all jobs and the total load on all machines. We show that the problems are polynomially solvable when the increasing rates are identical for all jobs; we propose a fully polynomial-time approximation scheme for the standard linear deteriorating function, where the objective function is to minimize the total load on all machines. We also consider the problem in which the processing time of a job is a simple linear increasing function of its starting time and each job has a delivery time. The objective is to find a schedule which minimizes the time by which all jobs are delivered, and we propose a fully polynomial-time approximation scheme to solve this problem.
\end{abstract}

Keywords Scheduling · Uniform machine $\cdot$ Linear deterioration $\cdot$ Fully polynomial time approximation scheme

\section{Introduction}

For most scheduling problems, the processing times of jobs are considered to be constant and independent of their starting time. However, this assumption is not appropriate for the modeling of many modern industrial processes, we often encounter

This work was supported by the National Natural Science Foundation of China (Nos. 11071142, 11201259), the Natural Science Foundation of Shan Dong Province (No. ZR2010AM034) and the Doctoral Fund of the Ministry of Education (No. 20123705120001).

J. Zou $(\bowtie) \cdot$ Y. Zhang

School of Management, Qufu Normal University, Rizhao, Shandong, China

e-mail: zoujuanjn@163.com

J. Zou · C. Miao

School of Mathematical Sciences, Qufu Normal University, Qufu, Shandong, China 
situations in which processing time increases over time, when the machines gradually lose efficiency. Such problems are generally known as scheduling with deterioration effects. Scheduling with linear deterioration was first considered by Browne and Yechiali [1] who assumed that the processing times of jobs are nondecreasing, start time dependent linear functions. They provided the optimal solution for the single machine when the objective is to minimize the makespan. In addition, they solved a special case when the objective function is to minimize the total weighted completion time. Mosheiov [2] considered simple linear deterioration where jobs have a fixed job-dependent growth rate but no basic processing time. He showed that most commonly applied performance criteria, such as the makespan, the total flow time, the total lateness, the sum of weighted completion time, the maximum lateness, the maximum tardiness, and the number of tardy jobs, remain polynomially solvable.

In the standard form of linear models, the actual processing time of job $J_{j}$ is given by $p_{j}=a_{j}+b_{j} t_{j}$, where $a_{j}, b_{j}$, and $t_{j}$ denote the basic processing time, the deteriorating rate, and the starting time of job $J_{j}$, respectively. Kuo and Yang [3] studied several parallel-machine scheduling problems in which the actual processing time of job $J_{j}$ is given by $p_{j}=a_{j}+b t_{j}$ or $p_{j}=a_{j}-b t_{j}$. The objectives are to minimize the total completion time of all jobs and the total load on all machines. They showed that the problems are polynomially solvable. Kononov [4] established the ordinary NP-completeness of $P 2\left|p_{j}=b_{j} t, r_{j}=t_{0}\right| C_{\max }$ and $P 2\left|p_{j}=b_{j} t, r_{j}=t_{0}\right| \sum C_{j}$. Mosheiov [5] studied multi-machine makespan minimization and total load minimization with linear deterioration, he proved that the two problems are NP-hard even for two machines. Liu et al. [6] considered $m$ uniform machine scheduling with linear deterioration to minimize the makespan, they proposed a fully polynomial-time approximation scheme for this problem. An extensive survey of different models and problems was provided by Alidaee and Womer [7]. Cheng et al. [8] presented an updated survey of the results on scheduling problems with time-dependent processing times. In addition, the concept of the learning effect and deteriorating jobs has been extensively studied. Lee [9] considered single-machine scheduling problem with deteriorating jobs and the learning effect, the objective was to minimize the makespan and the total completion time. He introduced the polynomial solutions for scheduling problems with the effects of learning and deterioration. But in the literature, there are only a few studies dealing with uniform parallel-machine scheduling problems under linear deterioration.

In this paper, we consider uniform-machine scheduling problems in which the actual processing time of job $J_{j}$ is given by $p_{j}=a_{j}+b t_{j}$. The objectives are to minimize the total completion time of all jobs and the total load on all machines. Following the three-field notation introduced by Graham et al. [10], the corresponding problems are denoted by $Q m\left|p_{j}=a_{j}+b t_{j}\right| \sum C_{j}$ and $Q m\left|p_{j}=a_{j}+b t_{j}\right| \sum C_{\max }^{i}$. We show that the two problems are polynomially solvable. For the standard linear deteriorating function, we denote the problem by $Q m\left|p_{j}=a_{j}+b_{j} t_{j}\right| \sum C_{\max }^{i}$. We propose a fully polynomial-time approximation scheme for this problem. We also consider the scheduling problem with simple linear deteriorating jobs on uniform parallel machine, each job $J_{j}$ has a delivery time $q_{j}$. The objective is to find a schedule which minimizes the time by which all jobs are delivered. Let $C_{j}$ denote the completion time of job $J_{j}$, thus the delivery completion time of a job $J_{j}$ is $C_{j}+q_{j}$, we will continue to 
use $L_{j}$ to represent $C_{j}+q_{j}$. The problem is denoted by $Q m\left|p_{j}=b_{j} t_{j}, r_{j}=t_{0}\right| L_{\max }$, we present a fully polynomial-time approximation scheme for it.

\section{Problem Description}

There are $n$ independent jobs $\mathcal{J}=\left\{J_{1}, J_{2}, \cdots, J_{n}\right\}$ to be processed on $m$ uniform parallel machines and each machine can process at most one job at a time. Preemption is not allowed. For the problems $Q m\left|p_{j}=a_{j}+b t_{j}\right| \sum C_{j}, Q m\left|p_{j}=a_{j}+b t_{j}\right| \sum C_{\max }^{i}$ and $Q m\left|p_{j}=a_{j}+b_{j} t_{j}\right| \sum C_{\max }^{i}$, we assume that all jobs are simultaneously available at time 0 and each job $J_{j}$ has a positive deteriorating rate $b$ or $b_{j}$. For the problem $Q m\left|p_{j}=b_{j} t_{j}, r_{j}=t_{0}\right| L_{\max }$, all the jobs are simultaneously available at time $t_{0}>0$. Each job $J_{j}$ has a positive deterioration rate $b_{j}$ and has a subsequent nonnegative delivery time $q_{j}$.

Let $M_{i}$ and $s_{i}$ denote the $i$ th machine in the system and its speed factor, respectively. Given a schedule, we use $n_{i}$ to denote the number of jobs scheduled on machine $M_{i}, J_{[i, r]}$ denotes a job when it is scheduled in position $r$ on machine $M_{i}$ in a sequence. Let $C_{[i, r]}, C_{\max }^{i}$, and $L_{[i, r]}$ denote the completion time of job $J_{[i, r]}$, the total load on machine $M_{i}$, and the delivery completion time of job $J_{[i, r]}$, respectively, for $i=1,2, \cdots, m, r=1,2, \cdots, n_{i}$, and $\sum_{i=1}^{m} n_{i}=n$.

For $Q m\left|p_{j}=a_{j}+b t_{j}\right| \sum C_{j}$ and $Q m\left|p_{j}=a_{j}+b t_{j}\right| \sum C_{\max }^{i}$, the completion time of each job scheduled on $M_{i}$ can be expressed as follows:

$$
\begin{aligned}
C_{[i, 1]} & =\frac{a_{[i, 1]}}{s_{i}}, \\
C_{[i, 2]} & =C_{[i, 1]}+\frac{a_{[i, 2]}+b C_{[i, 1]}}{s_{i}}=\frac{a_{[i, 2]}}{s_{i}}+\left(1+\frac{b}{s_{i}}\right) \frac{a_{[i, 1]}}{s_{i}}, \\
C_{[i, 3]} & =C_{[i, 2]}+\frac{a_{[i, 3]}+b C_{[i, 2]}}{s_{i}}=\frac{a_{[i, 3]}}{s_{i}}+\left(1+\frac{b}{s_{i}}\right) \frac{a_{[i, 2]}}{s_{i}}+\left(1+\frac{b}{s_{i}}\right)^{2} \frac{a_{[i, 1]}}{s_{i}}, \\
& \vdots \\
C_{[i, r]} & =C_{[i, r-1]}+\frac{a_{[i, r]}+b C_{[i, r-1]}}{s_{i}} \\
& =\frac{a_{[i, r]}}{s_{i}}+\left(1+\frac{b}{s_{i}}\right) \frac{a_{[i, r-1]}}{s_{i}}+\cdots+\left(1+\frac{b}{s_{i}}\right)^{r-1} \frac{a_{[i, 1]}}{s_{i}}, \\
C_{\max }^{i} & =C_{\left[i, n_{i}\right]}=\frac{a_{\left[i, n_{i}\right]}}{s_{i}}+\left(1+\frac{b}{s_{i}}\right) \frac{a_{\left[i, n_{i}-1\right]}}{s_{i}}+\cdots+\left(1+\frac{b}{s_{i}}\right)^{n_{i}-1} \frac{a_{[i, 1]}}{s_{i}} .
\end{aligned}
$$

The total completion time and the total load can be written as follows:

$$
\sum_{i=1}^{m} \sum_{r=1}^{n_{i}} C_{[i, r]}=\sum_{i=1}^{m} \sum_{r=1}^{n_{i}} \frac{1}{s_{i}}\left(1+\left(1+\frac{b}{s_{i}}\right)+\left(1+\frac{b}{s_{i}}\right)^{2}+\cdots+\left(1+\frac{b}{s_{i}}\right)^{n_{i}-r}\right) a_{[i, r]},
$$




$$
\sum_{i=1}^{m} C_{\max }^{i}=\sum_{i=1}^{m} \sum_{r=1}^{n_{i}} \frac{1}{s_{i}}\left(1+\frac{b}{s_{i}}\right)^{n_{i}-r} a_{[i, r]}
$$

For the problem $Q m\left|p_{j}=a_{j}+b_{j} t_{j}\right| \sum C_{\max }^{i}$, the total load on $M_{i}$ can be expressed as follows:

$C_{\max }^{i}=C_{\left[i, n_{i}\right]}=\frac{a_{\left[i, n_{i}\right]}}{s_{i}}+\sum_{j=1}^{n_{i}-1}\left(1+\frac{b_{\left[i, n_{i}\right]}}{s_{i}}\right)\left(1+\frac{b_{\left[i, n_{i}-1\right]}}{s_{i}}\right) \cdots\left(1+\frac{b_{[i, j+1]}}{s_{i}}\right) \frac{a_{[i, j]}}{s_{i}}$.

For the problem $Q m\left|p_{j}=b_{j} t_{j}, r_{j}=t_{0}\right| L_{\max }$, the delivery completion time of job $J_{[i, r]}$ can be denoted by $L_{[i, r]}=C_{[i, r]}+q_{[i, r]}=t_{0} \prod_{j=1}^{r}\left(1+\frac{b_{[i, j]}}{s_{i}}\right)+q_{[i, r]}$.

\section{Minimizing Total Completion Time and Minimizing Total Load}

\subsection{Polynomial Algorithms}

Lemma 1 Let $x_{i}$ and $y_{i}$ be two sequences of numbers, then the sum $\sum_{i} x_{i} y_{i}$ of products of the corresponding elements is the least if the sequences are monotonic in the opposite sense.

Proof The proof is obtained from [11].

Then, according to Lemma 1 and the expression form of the total completion time, we can construct a polynomial algorithm for the problem $Q m\left|p_{j}=a_{j}+b t_{j}\right| \sum C_{j}$.

\section{Algorithm 1}

Step 1 Select $n$ smallest numbers from the set $\left\{\frac{1}{s_{i}}, \frac{1}{s_{i}}+\frac{1}{s_{i}}\left(1+\frac{b}{s_{i}}\right), \frac{1}{s_{i}}+\frac{1}{s_{i}}\left(1+\frac{b}{s_{i}}\right)+\right.$ $\left.\frac{1}{s_{i}}\left(1+\frac{b}{s_{i}}\right)^{2}, \frac{1}{s_{i}}+\frac{1}{s_{i}}\left(1+\frac{b}{s_{i}}\right)+\frac{1}{s_{i}}\left(1+\frac{b}{s_{i}}\right)^{2}+\frac{1}{s_{i}}\left(1+\frac{b}{s_{i}}\right)^{3}, \cdots \mid i=1,2, \cdots, m\right\}$ and place these numbers in a nondecreasing sequence.

Step 2 Sort the jobs in the nonincreasing order of normal processing $a_{j}$ (i.e., $a_{n} \geqslant$ $\left.a_{n-1} \geqslant \cdots \geqslant a_{1}\right)$.

Step 3 Form a correspondence between these numbers and the jobs, if $J_{j}$ corresponds to $\frac{1}{s_{i}}+\frac{1}{s_{i}}\left(1+\frac{b}{s_{i}}\right)+\cdots+\frac{1}{s_{i}}\left(1+\frac{b}{s_{i}}\right)^{k-1}$, then schedule job $J_{j}$ on machine $M_{i}$ as the $k$ th last position.

Theorem 1 Algorithm 1 gives an optimal schedule for the problem $Q m \mid p_{j}=a_{j}+$ $b t_{j} \mid \sum C_{j}$ in $O(n \log n)$ time.

Proof Suppose that $\sigma^{*}$ is an optimal schedule which is different from the schedule $\sigma$ obtained by Algorithm 1. Let $k$ be the largest index such that job $J_{k}$ is scheduled on different processors in $\sigma^{*}$ and $\sigma$. Let job $J_{k}$ be scheduled on machine $M_{i}$ in $\sigma^{*}$ and $r_{i}$ denote the number of jobs scheduled to be processed after and including job $J_{k}$ on machine $M_{i}$ in $\sigma^{*}$. Let job $J_{k}$ be scheduled on machine $M_{j}$ in $\sigma$ and $r_{j}$ denote 
the number of jobs scheduled to be processed after and including job $J_{k}$ on machine $M_{j}$ in $\sigma$. Since $\sigma^{*}$ and $\sigma$ are identical for job indices greater than $k$ and Algorithm 1 assigned job $J_{k}$ to machine $M_{j}$, it follows that

$$
\frac{1}{s_{j}}+\frac{1}{s_{j}}\left(1+\frac{b}{s_{j}}\right)+\cdots+\frac{1}{s_{j}}\left(1+\frac{b}{s_{j}}\right)^{r_{j}-1} \leqslant \frac{1}{s_{i}}+\frac{1}{s_{i}}\left(1+\frac{b}{s_{i}}\right)+\cdots+\frac{1}{s_{i}}\left(1+\frac{b}{s_{i}}\right)^{r_{i}-1} .
$$

Let $J_{l}$ be the job at position $r_{j}$ in $\sigma^{*}$, by interchanging the position of job $J_{k}$ and $J_{l}$ in $\sigma^{*}$, the objective function of $\sigma^{*}$ changes by

$$
\begin{gathered}
\left(\sum_{r=0}^{r_{j}-1} \frac{1}{s_{j}}\left(1+\frac{b}{s_{j}}\right)^{r}\right) a_{k}+\left(\sum_{r=0}^{r_{i}-1} \frac{1}{s_{i}}\left(1+\frac{b}{s_{i}}\right)^{r}\right) a_{l} \\
-\left(\sum_{r=0}^{r_{j}-1} \frac{1}{s_{j}}\left(1+\frac{b}{s_{j}}\right)^{r}\right) a_{l}-\left(\sum_{r=0}^{r_{i}-1} \frac{1}{s_{i}}\left(1+\frac{b}{s_{i}}\right)^{r}\right) a_{k} \\
=\left(\sum_{r=0}^{r_{j}-1} \frac{1}{s_{j}}\left(1+\frac{b}{s_{j}}\right)^{r}-\sum_{r=0}^{r_{i}-1} \frac{1}{s_{i}}\left(1+\frac{b}{s_{i}}\right)^{r}\right)\left(a_{k}-a_{l}\right) \leqslant 0 .
\end{gathered}
$$

Based on the definition of $k$ and the ordering of job's normal processing time, it follows that $a_{k}-a_{l} \geqslant 0$. Then, a finite number of repetitions of this argument establishes that there exists an optimal schedule in which the jobs are sequenced by Algorithm 1.

Similarly, we give an optimal algorithm for the problem $Q m\left|p_{j}=a_{j}+b t_{j}\right| \sum C_{\max }^{i}$.

\section{Algorithm 2}

Step 1 Select $n$ smallest numbers from the set $\left\{\frac{1}{s_{i}}, \frac{1}{s_{i}}\left(1+\frac{b}{s_{i}}\right), \frac{1}{s_{i}}\left(1+\frac{b}{s_{i}}\right)^{2}, \cdots \mid i=\right.$ $1,2, \cdots, m\}$ and place these numbers in a nondecreasing sequence.

Step 2 Sort the jobs in the nonincreasing order of normal processing $a_{j}$.

Step 3 Form a correspondence between these numbers and the jobs, if $J_{j}$ corresponds to $\frac{1}{s_{i}}\left(1+\frac{b}{s_{i}}\right)^{k-1}$, then schedule job $J_{j}$ on machine $M_{i}$ as the $k$ th last position.

Theorem 2 Algorithm 2 gives an optimal schedule for the problem $Q m \mid p_{j}=a_{j}+$ $b t_{j} \mid \sum C_{\max }^{i}$, which can be solved in $O(n \log n)$ time.

Proof The proof is similar to that of Theorem 1.

\subsection{An FPTAS}

An algorithm $\mathcal{A}$ is a $\rho$-approximation $(\rho \geqslant 1)$ algorithm for a minimization problem if it produces a solution that is at most $\rho$ times that of the optimal one ( $\rho$ is also referred to as the worst-case ratio). A family of algorithms $\left\{\mathcal{A}_{\epsilon}: \epsilon>0\right\}$ is called a fully polynomial-time approximation scheme (FPTAS) if for each $\epsilon>0$ the algorithm 
$\mathcal{A}_{\epsilon}$ is a $(1+\epsilon)$-approximation algorithm running in polynomial time in the input size and $1 / \epsilon$. In the sequel, we assume $0<\epsilon \leqslant 1$.

Since the problem $P 2\left|p_{j}=b_{j} t_{j}, r_{j}=t_{0}\right| \sum C_{\max }^{i}$ has been proved to be ordinarily NP-hard by Mosheiov [5], $Q m\left|p_{j}=a_{j}+b_{j} t_{j}\right| \sum C_{\max }^{i}$ is at least NP-hard in the ordinary sense. In this section, we propose a fully polynomial-time approximation scheme for the scheduling problem $Q m\left|p_{j}=a_{j}+b_{j} t_{j}\right| \sum C_{\max }^{i}$. In order to use the procedure Partition proposed in Kovalyov and Kubiak [12] which requires that a function used within it be a nonnegative integer function, we first modify the original objective function $\sum C_{\max }^{i}$ to satisfy this restriction without affecting the sequence. The transformation method from the original objective function to a nonnegative integer function is given by the following scale procedure:

For any $i \in\{1, \cdots, m\}$ and $j \in\{1, \cdots, n\}$, define $\theta_{1}=\min \left\{\frac{a_{j}}{s_{i}}\right\}, \theta_{2}=\min \left\{1+\frac{b_{j}}{s_{i}}\right\}$. For simplicity, we suppose that $\theta_{1}$ and $\theta_{2}$ are finite decimals. Indeed, data are always obtained within some error range in industrial production. If the error range is set to be infinitesimal, the values will be real numbers. Find integers $\left\{l_{1}, l_{2}\right\} \in \mathbb{N}^{+}$such that $10^{l_{1}} \theta_{1} \in \mathbb{N}^{+}$and $10^{l_{2}} \theta_{2} \in \mathbb{N}^{+}$. Since

$$
C_{[i, r]} \frac{a_{[i, r]}}{s_{i}}+\sum_{j=1}^{r-1}\left(1+\frac{b_{[i, r]}}{s_{i}}\right)\left(1+\frac{b_{[i, r-1]}}{s_{i}}\right) \cdots\left(1+\frac{b_{[i, j+1]}}{s_{i}}\right) \frac{a_{[i, j]}}{s_{i}},
$$

$10^{l_{1}+(r-1) l_{2}} C_{[i, r]}$ can be verified as an integer. Then define $L=10^{l_{1}+n l_{2}}$, where $n$ is the total number of jobs, the transformed objective function can be expressed as $L \sum C_{\max }^{i}$. We use the new objective function instead of the original one in the following. Now our concerned problem can be denoted by $Q_{m} \mid p_{j}=a_{j}+$ $b_{j} t_{j} \mid L \sum C_{\max }^{i}$.

Gupta and Gupta [13] proved that the problem $1\left|p_{j}=a_{j}+b_{j} t_{j}\right| C_{\max }$ is solved by sequencing the jobs in nondecreasing order of $a_{j} / b_{j}$, which leads to the following lemma.

Lemma 2 For $Q m\left|p_{j}=a_{j}+b_{j} t_{j}\right| L \sum C_{\max }^{i}$, on each machine $M_{i}(i \in\{1, \cdots, m\})$ in an optimal solution, all the jobs are sequenced in the non-decreasing order of $\frac{a_{j}}{b_{j}}$.

Based on Lemma 2, it is natural to consider the jobs in nondecreasing order of $a_{j} / b_{j}$. So we index the jobs such that $a_{1} / b_{1} \leqslant a_{2} / b_{2} \leqslant \cdots \leqslant a_{n} / b_{n}$. We introduce variables $x_{j}, j=1,2, \cdots, n$, where $x_{j}=i$ if job $J_{j}$ is scheduled on machine $M_{i}, i=1,2, \cdots, m$. Let $X$ be the set of all vectors $x=\left(x_{1}, x_{2}, \cdots, x_{n}\right)$ with $x_{j} \in\{1,2, \cdots, m\}$, and $j=1,2, \cdots, n$. We define the following initial and recursive functions on $X$ :

Initial function: $F_{0}^{i}(x)=0, i=1, \cdots, m$.

Recursive function, for $j=1, \cdots, n$,

$$
\begin{aligned}
& F_{j}^{i}(x)=\frac{a_{j}}{s_{i}} 10^{l_{1}+j l_{2}}+F_{j-1}^{i}(x)\left(1+\frac{b_{j}}{s_{i}}\right) \cdot 10^{l_{2}}, \quad i=x_{j}, \\
& F_{j}^{i}(x)=F_{j-1}^{i}(x) \cdot 10^{l_{2}}, \quad i \neq x_{j},
\end{aligned}
$$




$$
G_{j}(x)=\sum_{i=1}^{m} F_{j}^{i}(x),
$$

where $F_{j}^{i}(x)$ is the magnified workload of machine $M_{i}$ for the jobs among $J_{1}, J_{2}, \cdots, J_{j}$.

Note that in the recursion functions, the workload of each machine is enlarged by a scale factor exactly $10^{l_{1}+n l_{2}}$. Therefore, $\sum_{i=1}^{m} F_{n}^{i}(x)$ is our desired value for a given $x$; the problem $Q_{m}\left|p_{j}=a_{j}+b_{j} t_{j}\right| L \sum C_{\max }^{i}$ reduces to the following minimization problem:

$$
\min G_{n}(x), \quad x \in X .
$$

First, we present the procedure Partition $(A, h, \delta)$ proposed by Kovalyov and $\mathrm{Ku}$ biak [12], where $A \subseteq X, h$ is a nonnegative integer function on $X$, and $0<\delta \leqslant 1$. This procedure partitions $A$ into disjoint subsets $A_{1}^{h}, A_{2}^{h}, \cdots, A_{k_{h}}^{h}$ such that $\mid h(x)-$ $h\left(x^{\prime}\right) \mid \leqslant \delta \min \left\{h(x), h\left(x^{\prime}\right)\right\}$ for any $x, x^{\prime}$ from the same subset $A_{j}^{h} j=1,2, \cdots, k_{h}$. The following description gives the details of Partition $(A, h, \delta)$.

Procedure Partition $(A, h, \delta)$

Arrange vectors $x \in A$ in the order $x^{(1)}, x^{(2)}, \cdots, x^{(|A|)}$ such that $0 \leqslant h\left(x^{(1)}\right) \leqslant$ $h\left(x^{(2)}\right) \leqslant \cdots \leqslant h\left(x^{(|A|)}\right)$.

Assign vectors $x^{(1)}, x^{(2)}, \cdots, x^{\left(i_{1}\right)}$ to the set $A_{1}^{h}$ until a certain $i_{1}$ is found such that $h\left(x^{\left(i_{1}\right)}\right) \leqslant(1+\delta) h\left(x^{(1)}\right)$ and $h\left(x^{\left(i_{1}+1\right)}\right)>(1+\delta) h\left(x^{(1)}\right)$. If such an $i_{1}$ does not exist, then set $A_{1}^{h}=A$ and stop.

Assign vectors $x^{\left(i_{1}+1\right)}, x^{\left(i_{1}+2\right)}, \cdots, x^{\left(i_{2}\right)}$ to the set $A_{2}^{h}$ until a certain $i_{2}$ is found such that $h\left(x^{\left(i_{2}\right)}\right) \leqslant(1+\delta) h\left(x^{\left(i_{1}+1\right)}\right)$ and $h\left(x^{\left(i_{2}+1\right)}\right)>(1+\delta) h\left(x^{\left(i_{1}+1\right)}\right)$. If such an $i_{2}$ does not exist, then set $A_{2}^{h}=A \backslash A_{1}^{h}$ and stop.

Continue the above construction until $x^{(|A|)}$ is included in $A_{k_{h}}^{h}$ for some $k_{h}$.

Obviously, procedure Partition $(A, h, \delta)$ requires $O(|A| \log |A|)$ operations to arrange the vectors of $A$ in nondecreasing order of $h(x)$ and $O(|A|)$ operations to provide a partition. The two properties of $\operatorname{Partition}(A, h, \delta)$ that will be used in the development of our FPTAS were presented in [12] as follows:

Lemma $3\left|h(x)-h\left(x^{\prime}\right)\right| \leqslant \delta \min \left\{h(x), h\left(x^{\prime}\right)\right\}$ for any $x, x^{\prime} \in A_{j}^{h}, j=1,2, \cdots, k_{h}$.

Lemma $4 k_{h} \leqslant \frac{\log h\left(x^{|A|}\right)}{\delta}+2$ if $h\left(x^{|A|}\right) \geqslant 1$.

Furthermore, we need the following lemma which was presented in [14].

Lemma 5 For any $0<x \leqslant 1$ and for any integer $n \geqslant 1,\left(1+\frac{x}{n}\right)^{n} \leqslant 1+2 x$ holds.

Motivated by the idea of Kovalyov and Kubiak [12], a formal description of the FPTAS $\mathcal{A}_{\epsilon}$ for problem $Q_{m}\left|p_{j}=a_{j}+b_{j} t_{j}\right| L \sum C_{\max }^{i}$ is given below. 


\section{Algorithm $\mathcal{A}_{\epsilon}$}

Step 1 (Initialization) Number the jobs so that $\frac{a_{1}}{b_{1}} \leqslant \frac{a_{2}}{b_{2}} \leqslant \cdots \leqslant \frac{a_{n}}{b_{n}}$. Set $Y_{0}=$ $\{(0,0, \cdots, 0)\}, F_{0}^{i}=0, i=1,2, \cdots, m$ and $j=1$.

Step 2 (Generation of $Y_{1}, Y_{2}, \cdots, Y_{n}$ ) For the set $Y_{j-1}$, we generate the set $Y_{j}^{\prime}$ by adding $k, k=1,2, \cdots, m$, in position $j$ of each vector from $Y_{j-1}$. Calculate the following for any $x \in Y_{j}^{\prime}$ :

$$
\begin{aligned}
F_{j}^{i}(x) & =\frac{a_{j}}{s_{i}} 10^{k_{1}+j k_{2}}+F_{j-1}^{i}(x)\left(1+\frac{b_{j}}{s_{i}}\right) \cdot 10^{k_{2}}, \quad i=x_{j}, \\
F_{j}^{i}(x) & =F_{j-1}^{i}(x) \cdot 10^{k_{2}}, \quad i \neq x_{j}, \\
G_{j}(x) & =\sum_{i=1}^{m} F_{j}^{i}(x) .
\end{aligned}
$$

If $j=n$, then set $Y_{n}=Y_{n}^{\prime}$ and go to Step 3 .

If $j<n$, then set $\delta=\frac{\epsilon}{2(n+1)}$ and perform the following computations:

Call Partition $\left(Y_{j}^{\prime}, F_{j}^{i}, \delta\right),(i=1,2, \cdots, m)$ to partition the set $Y_{j}^{\prime}$ into disjoint subsets $Y_{1}^{F^{i}}, Y_{2}^{F^{i}}, \cdots, Y_{F^{i}}^{F^{i}}$.

Divide the set $Y_{j}^{\prime}$ into disjoint subsets $Y_{c_{1}, \cdots, c_{m}}=Y_{c_{1}}^{F^{1}} \cap \cdots \cap Y_{c_{m}}^{F^{m}}, c_{1}=$ $1,2, \cdots, k_{F^{1}} ; \cdots ; c_{m}=1,2, \cdots, k_{F^{m}}$. For each nonempty subset $Y_{c_{1}, \cdots, c_{m}}$, choose a vector $x^{\left(c_{1}, \cdots, c_{m}\right)}$ such that

$$
G_{j}\left(x^{\left(c_{1}, \cdots, c_{m}\right)}\right)=\min \left\{G_{j}(x) \mid x \in Y_{c_{1}, \cdots, c_{m}}\right\} .
$$

Set $Y_{j}:=\left\{x^{\left(c_{1}, \cdots, c_{m}\right)}: Y_{c_{1}}^{F^{1}} \cap \cdots \cap Y_{c_{m}}^{F^{m}} \neq \emptyset\right.$, where $c_{1}=1,2, \cdots, k_{F^{1}} ; \cdots ; c_{m}=$ $\left.1,2, \cdots, k_{F^{m}}\right\}$ and set $j:=j+1$. Repeat Step 2 .

Step 3 (Solution) Select a vector $x^{0} \in Y_{n}$ such that

$$
G_{n}\left(x^{0}\right)=\min \left\{G_{n}(x) \mid x \in Y_{n}\right\} .
$$

Let $x^{*}=\left(x_{1}^{*}, x_{2}^{*}, \cdots, x_{n}^{*}\right)$ be an optimal solution for the problem $Q_{m} \mid p_{j}=a_{j}+$ $b_{j} t_{j} \mid L \sum C_{\max }^{i}$, write $x_{[j]}^{*}=\left(x_{1}^{*}, \cdots, x_{j}^{*}, 0, \cdots, 0\right)$ for $1 \leqslant j \leqslant n$.

We have the following theorem.

Theorem 3 For the problem $Q_{m}\left|p_{j}=a_{j}+b_{j} t_{j}\right| L \sum C_{\max }^{i}$, Algorithm $A_{\epsilon}$ finds $a$ solution $x^{0}$ such that $Q_{n}\left(x^{0}\right) \leqslant(1+\varepsilon) Q_{n}\left(x^{*}\right)$.

Proof Suppose that $x_{[j]}^{*}=\left(x_{1}^{*}, \cdots, x_{j}^{*}, 0, \cdots, 0\right) \in Y_{c_{1}, \cdots, c_{m}} \subseteq Y_{j}^{\prime}$ for some $j$ and $c_{1}, \cdots, c_{m}$. By the definition of Algorithm $\mathcal{A}_{\varepsilon}$, such $j$ always exists, for instance, $j=1$. Algorithm $\mathcal{A}_{\varepsilon}$ may not choose $x_{[j]}^{*}$ for further construction, however, a vector $x^{\left(c_{1}, \cdots, c_{m}\right)}$ is chosen instead of it. By Lemma 3 , we have

$$
\left|F_{j}^{i}\left(x_{[j]}^{*}\right)-F_{j}^{i}\left(x^{\left(c_{1}, \cdots, c_{m}\right)}\right)\right| \leqslant \delta F_{j}^{i}\left(x_{[j]}^{*}\right), \quad i=1,2, \cdots, m .
$$


Setting $\delta_{1}=\delta$, we consider the vectors $x_{[j+1]}^{*}=\left(x_{1}^{*}, \cdots, x_{j}^{*}, x_{j+1}^{*}, 0, \cdots, 0\right)$ and $\tilde{x}^{\left(c_{1}, \cdots, c_{m}\right)}=\left(x_{1}^{\left(c_{1}, \cdots, c_{m}\right)}, \cdots, x_{j}^{\left(c_{1}, \cdots, c_{m}\right)}, x_{j+1}^{*}, 0, \cdots, 0\right)$.

For $x_{j+1}^{*}=i, i \in\{1,2, \cdots, m\}$, we have

$$
\begin{aligned}
&\left|F_{j+1}^{i}\left(x_{[j+1]}^{*}\right)-F_{j+1}^{i}\left(\tilde{x}^{\left(c_{1}, \cdots, c_{m}\right)}\right)\right| \\
&=\mid \frac{a_{j+1}}{s_{i}} 10^{l_{1}+(j+1) l_{2}}+F_{j}^{i}\left(x_{[j]}^{*}\right)\left(1+\frac{b_{j+1}}{s_{i}}\right) 10^{l_{2}} \\
& \quad-\frac{a_{j+1}}{s_{i}} 10^{l_{1}+(j+1) l_{2}}-F_{j}^{i}\left(x^{\left(c_{1}, \cdots, c_{m}\right)}\right)\left(1+\frac{b_{j+1}}{s_{i}}\right) 10^{l_{2}} \mid \\
&=\left(1+\frac{b_{j+1}}{s_{i}}\right) 10^{l_{2}}\left|F_{j}^{i}\left(x_{[j]}^{*}\right)-F_{j}^{i}\left(x^{\left(c_{1}, \cdots, c_{m}\right)}\right)\right| \\
& \leqslant\left(1+\frac{b_{j+1}}{s_{i}}\right) 10^{l_{2}} \delta F_{j}^{i}\left(x_{[j]}^{*}\right) \\
& \leqslant \delta_{1} F_{j+1}^{i}\left(x_{[j+1]}^{*}\right) .
\end{aligned}
$$

Similarly, for $x_{j+1}^{*} \neq i$, we have

$$
\left|F_{j+1}^{i}\left(x_{[j+1]}^{*}\right)-F_{j+1}^{i}\left(\tilde{x}^{\left(c_{1}, \cdots, c_{m}\right)}\right)\right| \leqslant \delta_{1} F_{j+1}^{i}\left(x_{[j+1]}^{*}\right) .
$$

As a consequence, we have

$$
\left|F_{j+1}^{i}\left(x_{[j+1]}^{*}\right)-F_{j+1}^{i}\left(\tilde{x}^{\left(c_{1}, \cdots, c_{m}\right)}\right)\right| \leqslant \delta_{1} F_{j+1}^{i}\left(x_{[j+1]}^{*}\right), \quad i=1,2, \cdots, m .
$$

This leads to

$$
F_{j+1}^{i}\left(\tilde{x}^{\left(c_{1}, \cdots, c_{m}\right)}\right) \leqslant\left(1+\delta_{1}\right) F_{j+1}^{i}\left(x_{[j+1]}^{*}\right), \quad i=1,2, \cdots, m .
$$

Assume that $\tilde{x}^{\left(c_{1}, \cdots, c_{m}\right)} \in Y_{b_{1}, \cdots, b_{m}} \subseteq Y_{j+1}^{\prime}$ and Algorithm $\mathcal{A}_{\epsilon}$ chooses $x^{b_{1}, \cdots, b_{m}} \in$ $Y_{b_{1}, \cdots, b_{m}}$ instead of $\tilde{x}^{\left(c_{1}, \cdots, c_{m}\right)}$ in the $(j+1)$ th iteration. From (2) and by Lemma 3 , for $i=1,2, \cdots, m$, we have

$$
\begin{aligned}
\left|F_{j+1}^{i}\left(\tilde{x}^{\left(c_{1}, \cdots, c_{m}\right)}\right)-F_{j+1}^{i}\left(x^{b_{1}, \cdots, b_{m}}\right)\right| & \leqslant \delta F_{j+1}^{i}\left(\tilde{x}^{\left(c_{1}, \cdots, c_{m}\right)}\right) \\
& \leqslant \delta\left(1+\delta_{1}\right) F_{j+1}^{i}\left(x_{[j+1]}^{*}\right) .
\end{aligned}
$$

From (1) and (3), for $i=1,2, \cdots, m$, we obtain

$$
\begin{aligned}
& \left|F_{j+1}^{i}\left(x_{[j+1]}^{*}\right)-F_{j+1}^{i}\left(x^{b_{1}, \cdots, b_{m}}\right)\right| \\
& \quad \leqslant\left|F_{j+1}^{i}\left(x_{[j+1]}^{*}\right)-F_{j+1}^{i}\left(\tilde{x}^{\left(c_{1}, \cdots, c_{m}\right)}\right)\right|+\left|F_{j+1}^{i}\left(\tilde{x}^{\left(c_{1}, \cdots, c_{m}\right)}\right)-F_{j+1}^{i}\left(x^{b_{1}, \cdots, b_{m}}\right)\right| \\
& \quad \leqslant\left(\delta_{1}+\delta\left(1+\delta_{1}\right)\right) F_{j+1}^{i}\left(x_{[j+1]}^{*}\right) \\
& \quad=\left(\delta+\delta_{1}(1+\delta)\right) F_{j+1}^{i}\left(x_{[j+1]}^{*}\right) .
\end{aligned}
$$


Set $\delta_{l}=\delta+\delta_{l-1}(1+\delta), l=2,3, \cdots, n-j+1$. From (4), we have

$$
\left|F_{j+1}^{i}\left(x_{[j+1]}^{*}\right)-F_{j+1}^{i}\left(x^{b_{1}, \cdots, b_{m}}\right)\right| \leqslant \delta_{2} F_{j+1}^{i}\left(x_{[j+1]}^{*}\right), \quad i=1,2, \cdots, m .
$$

By repeating the above argument for $j+2, \cdots, n$, we eventually show that there exists an $x^{\prime} \in Y_{n}$ such that

$$
\left|F_{n}^{i}\left(x^{*}\right)-F_{n}^{i}\left(x^{\prime}\right)\right| \leqslant \delta_{n-j+1} F_{n}^{i}\left(x^{*}\right), \quad i=1,2, \cdots, m .
$$

By Lemma 5, we have

$$
\delta_{n-j+1} \leqslant \delta \sum_{j=0}^{n}(1+\delta)^{j}=(1+\delta)^{n+1}-1 \leqslant\left(1+\frac{\epsilon}{2(n+1)}\right)^{n+1}-1 \leqslant \epsilon .
$$

Therefore,

$$
F_{n}^{i}\left(x^{\prime}\right) \leqslant\left(1+\delta_{n-j+1}\right) F_{n}^{i}\left(x^{*}\right) \leqslant(1+\epsilon) F_{n}^{i}\left(x^{*}\right), \quad i=1,2, \cdots, m .
$$

From (5), we have

$$
G_{n}\left(x^{\prime}\right)=\sum_{i=1}^{m} F_{n}^{i}\left(x^{\prime}\right) \leqslant(1+\epsilon) \sum_{i=1}^{m} F_{n}^{i}\left(x^{*}\right)=(1+\epsilon) G_{n}\left(x^{*}\right) .
$$

By Step 3 of Algorithm $\mathcal{A}_{\epsilon}$, a vector $x^{0}$ will be chosen such that

$$
G_{n}\left(x^{0}\right) \leqslant G_{n}\left(x^{\prime}\right) \leqslant(1+\epsilon) G_{n}\left(x^{*}\right) .
$$

This completes the proof.

Define $L=\log \max \left\{n, \frac{1}{\epsilon}, A_{\max }, 1+B_{\max }, 10^{l_{1}}, 10^{l_{2}}\right\}$, where $A_{\max }=\max \left\{\frac{a_{j}}{s_{i}}\right\}$, $B_{\max }=\max \left\{\frac{b_{j}}{s_{i}}\right\}, i=1, \cdots, m ; j=1, \cdots, n$. We have the following theorem.

Theorem 4 Algorithm $\mathcal{A}_{\epsilon}$ requires $O\left(\frac{n^{2 m+1} L^{m+1}}{\epsilon^{m}}\right)$ time.

Proof The time complexity of Algorithm $\mathcal{A}_{\epsilon}$ can be established by noting that the most time-consuming operation of iteration $j$ of Step 2 is a call of procedure Partition, which requires $O\left(\left|Y_{j}^{\prime}\right| \log \left|Y_{j}^{\prime}\right|\right)$ time to complete. To estimate $\left|Y_{j}^{\prime}\right|$, recall that

$$
\left|Y_{j+1}^{\prime}\right| \leqslant m\left|Y_{j}\right| \leqslant m k_{F^{1}} k_{F^{2}} \cdots k_{F^{m}} .
$$

By Lemma 4, we have

$$
\begin{aligned}
k_{F^{i}} & \leqslant \frac{2(n+1) \log \left(10^{l_{1}+n l_{2}} n A_{\max }\left(1+B_{\max }\right)^{n}\right)}{\epsilon}+2 \\
& \leqslant \frac{2(n+1)(2 n+3) L}{\epsilon}+2, \quad i=1,2, \cdots, m .
\end{aligned}
$$


Thus $\left|Y_{j}^{\prime}\right|=O\left(\frac{n^{2 m} L^{m}}{\epsilon^{m}}\right)$ and $\left|Y_{j}^{\prime}\right| \log \left|Y_{j}^{\prime}\right|=O\left(\frac{n^{2 m} L^{m+1}}{\epsilon^{m}}\right)$. There are at most $n$ iterations; therefore, Algorithm $\mathcal{A}_{\epsilon}$ runs in $O\left(\frac{n^{2 m+1} L^{m+1}}{\epsilon^{m}}\right)$ time.

\section{Minimizing the Maximum Delivery-Completion Time}

\subsection{NP-Hardness}

Theorem 5 The problem $Q m\left|p_{j}=b_{j} t_{j}, r_{j}=t_{0}\right| L_{\max }$ is NP-hard.

Proof If all delivery times are zero, the problem $P m\left|p_{j}=b_{j} t_{j}, r_{j}=t_{0}\right| L_{\max }$ is equivalent to the problem $P m\left|p_{j}=b_{j} t_{j}, r_{j}=t_{0}\right| C_{\max }$. Since $P m \mid p_{j}=b_{j} t_{j}, r_{j}=$ $t_{0} \mid C_{\max }$ is ordinarily NP-hard according to Kononov [4], $Q m \mid p_{j}=b_{j} t_{j}, r_{j}=$ $t_{0} \mid L_{\max }$ is at least NP-hard in the ordinary sense.

\subsection{An FPTAS}

In this section, we derive a fully polynomial-time approximation scheme for the NPhard problem $Q m\left|p_{j}=b_{j} t_{j}, r_{j}=t_{0}\right| L_{\max }$. This is done by applying the "roundingthe-input-data" technique of Sahni [15] to a given problem instance. The definition of the modified deteriorating rates involves a geometric rounding technique developed by Sengupta [16]. And the rounding technique is stated as follows:

For any $\epsilon^{\prime}>0$ and $x \geqslant 1$, if $\left(1+\epsilon^{\prime}\right)^{k-1}<x<\left(1+\epsilon^{\prime}\right)^{k}$, then we define $\lceil x\rceil_{\epsilon^{\prime}}=$ $\left(1+\epsilon^{\prime}\right)^{k},\lfloor x\rfloor_{\epsilon^{\prime}}=\left(1+\epsilon^{\prime}\right)^{k-1}$. If $x$ is an exact power of $\left(1+\epsilon^{\prime}\right)$, then $\lceil x\rceil_{\epsilon^{\prime}}=$ $\lfloor x\rfloor_{\epsilon^{\prime}}=x$. Note that $\lceil x\rceil_{\epsilon^{\prime}} \leqslant\left(1+\epsilon^{\prime}\right) x$ for any $x \geqslant 1$.

For any $0<\epsilon \leqslant 1$, we define the modified deteriorating rate of job $J_{j}$ as $b_{j}^{\prime}=$ $s_{i}\left(\left\lceil 1+\frac{b_{j}}{s_{i}}\right\rceil_{\epsilon^{\prime}}-1\right)$, where $\epsilon^{\prime}=\frac{\epsilon}{2 n}$, for $i=1, \cdots, m$ and $j=1, \cdots, n$. Let $\beta_{j i}$ denote the exponent of $1+\frac{b_{j}^{\prime}}{s_{i}}$, i.e., $1+\frac{b_{j}^{\prime}}{s_{i}}=\left\lceil 1+\frac{b_{j}}{s_{i}}\right\rceil_{\epsilon^{\prime}}=\left(1+\epsilon^{\prime}\right)^{\beta_{j i}}$, then $\beta_{j i}=$ $\frac{\log \left\lceil 1+\frac{b_{j}}{s_{i}}\right\rceil_{\epsilon^{\prime}}}{\log \left(1+\epsilon^{\prime}\right)}=O\left(\frac{n \log \left(1+\frac{b_{j}}{s_{i}}\right)}{\epsilon}\right)$.

Theorem 6 For any $0<\epsilon \leqslant 1$, the optimal objective function value for $Q_{m} \mid p_{j}=$ $b_{j} t_{j}, r_{j}=t_{0} \mid L_{\max }$ under the modified deteriorating rates is at most $(1+\epsilon)$ times the optimal value for the same problem under the original deteriorating rates.

Proof Let $\sigma$ be an arbitrary feasible schedule. Consider an arbitrary job $J_{j}$ scheduled in position $r$ on machine $M_{i}, 1 \leqslant i \leqslant m$. We denote by $C_{j}$ the completion time of $J_{j}$ under the original deteriorating rates, and by $C_{j}^{\prime}\left(C_{[i, r]}^{\prime}\right)$ the completion time of $J_{j}$ under the modified deteriorating rates in $\sigma$. By the definition of the modified deteriorating rate $b_{j}^{\prime}$, we have

$$
1+\frac{b_{j}^{\prime}}{s_{i}}=\left\lceil 1+\frac{b_{j}}{s_{i}}\right\rceil_{\epsilon^{\prime}} \leqslant\left(1+\epsilon^{\prime}\right)\left(1+\frac{b_{j}}{s_{i}}\right) .
$$


This yields

$$
C_{j}^{\prime}=C_{[i, r]}^{\prime}=t_{0} \prod_{l=1}^{r}\left(1+\frac{b_{[i, l]}^{\prime}}{s_{i}}\right) \leqslant t_{0}\left(1+\epsilon^{\prime}\right)^{r} \prod_{l=1}^{r}\left(1+\frac{b_{[i, l]}}{s_{i}}\right) \leqslant\left(1+\epsilon^{\prime}\right)^{n} C_{j} .
$$

Since $\left(1+\epsilon^{\prime}\right)^{n}=\left(1+\frac{\epsilon}{2 n}\right)^{n} \leqslant 1+\epsilon$ for $0<\epsilon \leqslant 2$,

$$
L_{j}^{\prime}=C_{j}^{\prime}+q_{j} \leqslant\left(1+\epsilon^{\prime}\right)^{n}\left(C_{j}+q_{j}\right) \leqslant(1+\epsilon)\left(C_{j}+q_{j}\right)=(1+\epsilon) L_{j} .
$$

As a consequence, we have

$$
L_{\max }^{\prime}=\max \left\{L_{j}^{\prime}\right\} \leqslant(1+\epsilon) \max \left\{L_{j}\right\}=(1+\epsilon) L_{\max }(\sigma) .
$$

Since the above inequality is valid for any feasible schedule $\sigma$, the results holds.

Lemma 6 For a single machine problem $1\left|p_{j}=b_{j} t, r_{j}=t_{0}\right| L_{\max }$, sorting the jobs by nonincreasing delivery times yields an optimum schedule.

Proof Consider an optimal schedule $\sigma^{*}$. If $\sigma^{*}$ contains jobs that are not sequenced in nonincreasing order of delivery times, then there exists a pair of adjacent jobs $J_{i}, J_{j}$ such that job $J_{i}$ is followed by job $J_{j}$ but $q_{i}<q_{j}$. Assuming that job $J_{i}$ starts at time $S$, we have

$$
L_{i}\left(\sigma^{*}\right)=S+b_{i} S+q_{i}, \quad L_{j}\left(\sigma^{*}\right)=S+b_{i} S+b_{j}\left(S+b_{i} S\right)+q_{j} .
$$

Consider a schedule $\sigma$ which is obtained from $\sigma^{*}$ by interchanging jobs $J_{i}$ and $J_{j}$. Under $\sigma$, we have

$$
L_{j}(\sigma)=S+b_{j} S+q_{j}, \quad L_{i}(\sigma)=S+b_{j} S+b_{i}\left(S+b_{j} S\right)+q_{i},
$$

then we obtain that

$$
L_{j}\left(\sigma^{*}\right)>L_{j}(\sigma), \quad L_{j}\left(\sigma^{*}\right)>L_{i}(\sigma) .
$$

This contradicts the optimality of $\sigma^{*}$ since all other completion times are unchanged. It follows that sorting the jobs by nonincreasing delivery times yields an optimum schedule for a single machine.

Corollary 1 There exists an optimal job sequence for $Q m\left|p_{j}=b_{j} t_{j}, r_{j}=t_{0}\right| L_{\max }$ such that on each machine the jobs are sequenced in nonincreasing order of delivery times.

As a result of Theorem 6 , with $(1+\epsilon)$ loss, we can design a dynamic program for the problem $Q_{m}\left|p_{j}=b_{j} t, r_{j}=t_{0}\right| L_{\max }$ under the modified deteriorating rates. Based on Corollary 1 , it is natural to consider the jobs in nonincreasing order of $q_{j}$. Let us index the jobs such that $q_{1} \geqslant q_{2} \geqslant \cdots \geqslant q_{n}$. The dynamic programming algorithm is stated as follows: 
Algorithm DP Let $\phi_{j}\left(\gamma_{1}, \gamma_{2}, \cdots, \gamma_{m}\right)$ denote the optimal value when the jobs in consideration are $J_{1}, J_{2}, \cdots, J_{j}$, and the maximum completion time of jobs on machine $M_{k}$ is $t_{0}\left(1+\epsilon^{\prime}\right)^{\gamma_{k}}$ for $k=1,2, \cdots, m$.

Step 1 (Initialization)

$$
\phi_{0}\left(\gamma_{1}, \gamma_{2}, \cdots, \gamma_{m}\right)= \begin{cases}t_{0}, & \text { if } \gamma_{1}=\cdots=\gamma_{m}=0, \\ +\infty, & \text { otherwise. }\end{cases}
$$

Step 2 (Recursion)

$$
\begin{aligned}
& \phi_{j}\left(\gamma_{1}, \gamma_{2}, \cdots, \gamma_{m}\right) \\
& \quad=\min _{1 \leqslant k \leqslant m}\left\{\max \left\{\phi_{j-1}\left(\gamma_{1}, \cdots, \gamma_{k}-\beta_{j k}, \cdots, \gamma_{m}\right), t_{0}\left(1+\epsilon^{\prime}\right)^{\gamma_{k}}+q_{j}\right\}\right\} .
\end{aligned}
$$

If $j=n$, go to Step 3. Otherwise, set $j:=j+1$ and repeat Step 2.

Step 3 (Output) The optimal value is determined by

$$
\min \left\{\phi_{n}\left(\gamma_{1}, \gamma_{2}, \cdots, \gamma_{m}\right) \mid 0 \leqslant \gamma_{k} \leqslant \sum_{j=1}^{n} \beta_{j k}, 1 \leqslant k \leqslant m\right\} .
$$

The corresponding optimal schedule can be found by backtracking.

Some remarks should be made about Algorithm DP. In Step 2, we assume that $J_{j}$ is scheduled on machine $M_{k}, 1 \leqslant k \leqslant m$. For the jobs $J_{1}, J_{2}, \cdots, J_{j-1}$, the maximum completion time of jobs on machine $M_{i}$ must be $t_{0}\left(1+\epsilon^{\prime}\right)^{\gamma_{i}}$, for $i=1, \cdots, k-1, k+1, \cdots, m$, and be $t_{0}\left(1+\epsilon^{\prime}\right)^{\gamma_{k}-\beta_{j k}}$ on machine $M_{k}$. Hence the delivery completion time of $J_{j}$ is compared with the maximum delivery completion times of the jobs that have been scheduled earlier.

Let $L=\log \left(1+\frac{b_{\max }}{s_{\min }}\right)$, where $\frac{b_{\max }}{s_{\min }}=\max \left\{\frac{b_{j}}{s_{i}}: 1 \leqslant j \leqslant n, 1 \leqslant i \leqslant m\right\}$. We have the following theorem.

Theorem 7 There exists an FPTAS for the problem $Q m\left|p_{j}=b_{j} t, r_{j}=t_{0}\right| L_{\max }$ which runs in $O\left(n^{2 m-1} L^{m-1} / \epsilon^{m-1}\right)$ time.

Proof In the dynamic programming recursion, we have $n$ states for $j$, and at most $\sum_{j=1}^{n} \beta_{j k}$ states for each $\gamma_{k}, 1 \leqslant k \leqslant m$, where only $m-1$ of the values $\gamma_{1}, \gamma_{2}, \cdots, \gamma_{m}$ are independent. So the total complexity is $O\left(n\left(\sum_{j=1}^{n} \beta_{j k}\right)^{m-1}\right)=$ $O\left(n^{2 m-1} L^{m-1} / \epsilon^{m-1}\right)$.

\section{Conclusion}

In this paper, we focus on uniform parallel machine scheduling problems with time dependent processing times. The actual processing time of a job is a linear increasing function of its starting time. The objectives are to minimize the total completion time of all jobs and the total load on all machines. When all jobs are associated with a common deteriorating rate $b$, we provide an optimal $O(n \log n)$ time algorithm 
for them. We also presented fully polynomial-time approximation schemes for the problems $Q m\left|p_{j}=a_{j}+b_{j} t_{j}\right| \sum C_{\max }^{i}$ and $Q m\left|p_{j}=b_{j} t_{j}, r_{j}=t_{0}\right| L_{\max }$. For future research, it would be interesting to focus on scheduling deteriorating jobs with other objectives.

Acknowledgements We thank the two anonymous reviewers for their helpful and detailed comments on an earlier version of our paper.

\section{References}

[1] Browne, S., Yechiali, U.: Scheduling deteriorating jobs on a single processor. Oper. Res. 38, 495-498 (1990)

[2] Mosheiov, G.: Scheduling jobs under simple linear deterioration. Comput. Oper. Res. 2(1), 653-659 (1994)

[3] Kuo, W.H., Yang, D.L.: Parallel-machine scheduling with time dependent processing times. Theor. Comput. Sci. 393, 204-210 (2008)

[4] Kononov, A.: Scheduling problems with linear increasing processing times. Oper. Res. Proc. 1996, 208-212 (1997)

[5] Mosheiov, G.: Multi-machine scheduling with linear deterioration. Inf. Syst. Oper. Res. 36, 205-214 (1998)

[6] Liu, M., Zheng, F.F., Chu, C.B.: An FPTAS for uniform machine scheduling to minimize makespan with linear deterioration. J. Comb. Optim. 23, 483-492 (2012)

[7] Alidaee, B., Womer, N.K.: Scheduling with time dependent processing times: review and extension. J. Oper. Res. Soc. 50, 711-720 (1999)

[8] Cheng, T.C.E., Ding, Q., Lin, B.M.T.: A concise survey of scheduling with time-dependent processing times. Eur. J. Oper. Res. 152, 1-13 (2004)

[9] Lee, W.C.: A note on deteriorating jobs and learning in single-machine scheduling problems. Int. J. Bus. Econ. 3, 205-214 (2004)

[10] Graham, R.L., Lawler, E.L., Lenstra, J.K., Rinnooy Kan, A.H.G.: Optimization and approximation in deterministic sequencing and scheduling: a survey. Ann. Discrete Math. 5, 287-326 (1979)

[11] Hardy, G.H., Littlewood, J.E., Polya, G.: Inequalities. Cambridge University Press, London (1967)

[12] Kovalyov, M.Y., Kubiak, W.: A fully polynomial approximation scheme for minimizing makespan of deteriorating jobs. J. Heuristics 3, 287-297 (1998)

[13] Gupta, J.N.D., Gupta, S.K.: Single facility scheduling with nonlinear processing times. Comput. Ind. Eng. 14, 387-394 (1988)

[14] Engels, D.W., Karger, D.R., Kolliopoulos, S.G., Sengupta, S., Uma, R.N., Wein, J.: Techniques for scheduling with rejection. J. Algorithms 49, 175-191 (2003)

[15] Sahni, S.: Algorithms for scheduling independent tasks. J. ACM 23, 116-127 (1976)

[16] Sengupta, S.: Algorithms and approximation schemes for minimum lateness/tardiness scheduling with rejection. Lecture Notes in Computer Science, vol. 2748. pp. 79-90 (2003) 